\title{
TESTING THE EVOLUTIONARY SEQUENCE BETWEEN HIDDEN BROAD LINE REGION (HBLR) AND NON-HBLR SEYFERT 2 GALAXIES WITH THE 4000-Å BREAK STRENGTHS
}

\author{
PO-CHIEH YU*ं \\ Graduate Institute of Astronomy, National Central University, Chung-Li 32054, Taiwan \\ E-mail: pcyudastro.ncu.edu.tw
}

\section{KUI-YUN HUANG}

Institute of Astronomy and Astrophysics, Academia Sinica, P.O. Box 23-141, Taipei 10617,

Taiwan

E-mail: Ljhuangdasiaa.sinica.edu.tw

\section{CHORNG-YUAN HWANG}

Graduate Institute of Astronomy, National Central University, Chung-Li 32054, Taiwan

E-mail: hwangcydastro.ncu.edu.tw

\section{YOUICHI OHYAMA}

Institute of Astronomy and Astrophysics, Academia Sinica, P.O. Box 23-141, Taipei 10617,

Taiwan

E-mail: bhyamadasiaa.sinica.edu.tw

We compared the 4000 Angstrom break (D4000) strength of hidden broad line regions (HBLR) and non-HBLR Seyfert 2 galaxies. Previous studies showed that only about $40 \%-45 \%$ of Seyfert 2 galaxies show HBLR. The reason of the non-detection of HBLR in some Seyfert 2 galaxies is still under debated. Our results show that the D4000 strength of the non-HBLR Seyfert 2 galaxies is larger than that of HBLR ones, implying that the non-HBLR Seyfert 2 galaxies have older stellar population in the nuclear regions. This suggests that there might be an evolutionary connection between the non-HBLR and HBLR Seyfert 2 galaxies. The gas around the nucleus of the non-HBLR Seyfert 2 galaxies might have diminished in the older age, which would cause low accretion rates. When the accretion rate is below some thresholds, the broad line regions could disappear.

Nuclei of Seyfert galaxies and QSOs - Central engine \& conditions of star formation November 6-8, 2012

Max-Planck Institute für Radioastronomie (MPIfR), Bonn, Germany

\footnotetext{
* Speaker.

$\dagger$ This work was partially supported by the National Science Council of Taiwan (grants NSC 99-2112-M-008-014MY3 (C.-Y.H.), NSC 100-2119-M-008-011-MY3 (C.-Y.H.), NSC 99-2112-M-001-002-MY3 (K.Y.H.), and NSC 1002112-M-001-001-MY3 (Y.O.))
} 


\section{INTRODUCTION}

Seyfert galaxies are radio-quiet active galactic nuclei (AGNs) and further divided into two subtypes, Seyfert 1 and Seyfert 2 galaxies. According to the unification model[1], Seyfert 2 galaxies are suggested to be the same objects as Seyfert 1 but viewed from an edge-on direction. The broad-line region (BLR) of Seyfert 2 galaxies is obscured by a dusty torus and thus only forbidden emission lines can be observed. Photons from BLRs could be scattered by electrons and thus that might be detectable by the polarimetry in Seyfert 2 galaxies. Previous studies indicated that some Seyfert 2 galaxies did show polarized broad permitted emission lines. The discovery of the hidden broad-line region (HBLR) further supported the unification model.

However, previous polarimetry survey showed that only about $40 \%-50 \%$ of Seyfert 2 galaxies exhibit HBLRs[2]. The non-detection of the HBLRs in some Seyfert 2 galaxies challenges the unification model. The reason causing the non-detection of HBLRs is still under debated. Several possibilities have been proposed: (1) Based on the ratio of $f_{25} / f_{60}$, Heisler et al. (1997)[3] suggested that the detectability of the HBLR in Seyfert 2 galaxies is related to the orientation of the torus. (2) Some evolutionary processes might be at work between HBLR and non-HBLR Seyfert 2 galaxies[2][4]. (3) Zhang \& Wang (2006)[5] found that non-HBLR Seyfert 2 galaxies and narrow line Seyfert 1 galaxies (NLS1s) have similar distribution of black hole masses, accretion rate and the ratio of $f_{25} / f_{60}$. Non-HBLR Seyfert 2 galaxies were thus suggested to be the counterparts of NLS1s at an edge-on orientation. (4) Non-HBLR Seyfert 2 galaxies might be powered by nuclear starbursts rather than accretion onto the central black hole[6]. (5) Shu et al. (2007)[7] showed that the the visibility of polarized broad lines might be affected by nuclear activity and obscuration. (6) Some Seyfert 2 galaxies could intrinsically lack broad line regions[8]. (8) Some Seyfert 2 galaxies might be deficient in scattering material.

Yu \& Hwang (2011)[9] discovered that the [N II]/H $\alpha$ ratios of non-HBLR Seyfert 2 galaxies are higher than those of HBLR Seyfert 2 galaxies. They suggested that the difference could be caused by different evolutionary stages of starburst activities in non-HBLR and HBLR Seyfert 2 galaxies. We estimate the 4000- $\AA$ break $\left(D_{n}(4000)\right)$ strengths[10] of these galaxies to further investigate the age of the stellar population around the nuclei of the HBLR and non-HBLR Seyfert 2 galaxies. The $D_{n}(4000)$ strength is an indicator that provides reliable estimates for the ages of stellar populations[11]. The elements contributing to the opacity are usually ionized metals. $D_{n}(4000)$ is caused by absorption of high energy radiation from metals in stellar atmospheres. The $D_{n}(4000)$ strength is thus small for young, blue stars and large for old, metal-rich ones .

\section{OBSERVATIONS}

Nine Seyfert 2 galaxies were observed using a 1.8-m telescope, with a low-resolution spectrograph and a long-slit at the Bohyunsan Optical Astronomy Observatory (BOAO) in South Korea. The observations were carried out over three multi-day periods: 2011 October 27-31; 2012 January 9-12; and 2012 May 3-7. Approximately 5.5 observable nights had a seeing of 2" - 3". Using a 150 groove per mm grating and a slit width of $2.9^{\prime \prime}$, we covered a wavelength range of $3500-7500 \AA$ at a spectral resolution of $\lambda / \delta \lambda \sim 366$. Using the IRAF package, we then follow the standard data reduction procedure, i.e., flat-fielding, wavelength calibration, and flux calibration. The spectra of 
16 additional Seyfert 2 galaxies were also obtained from the Sloan Digital Sky Survey (SDSS), as well as the spectrum of NGC 3079 from the NASA/IPAC Extragalactic Database (NED). We estimated the $D_{n}(4000)$ strengths of 10 HBLR and 16 non-HBLR Seyfert 2 galaxies.

\section{RESULTS AND DISCUSSION}

The optical spectra of the HBLR Seyfert 2 galaxies showed weak stellar absorption lines. However, the spectra of the non-HBLR Seyfert 2 galaxies showed strong 4000- $\AA$ break and stellar absorption lines, such as the G band near $4300 \AA$ and the magnesium features near $5200 \AA$. We then estimate the $D_{n}(4000)$ strength to conduct a quantitative comparison. The $D_{n}(4000)$ index was originally defined by Bruzual (1983)[12] and redefined by Balgoh et al.(1999)[10] to reduce the reddening effects:

$$
D_{n}(4000)=\frac{\int_{4000}^{4100} f_{\lambda} d \lambda}{\int_{3850}^{3950} f_{\lambda} d \lambda} .
$$

We removed the contamination of emission lines in our measurements of the $D_{n}(4000)$ strengths. Figure 1 shows the $D_{n}(4000)$ strengths and the spectral index Fe5270 of the 10 HBLR and 16 nonHBLR Seyfert 2 galaxies. Both the Kolmogorov-Smirnov test $(P=99.1 \%)$ and Studentąs $T$-test $(P=99.6 \%)$ show that the $D_{n}(4000)$ distribution of the HBLR and non-HBLR Seyfert 2 galaxies are significantly different, indicating they have different stellar populations. The $D_{n}(4000)$ can be separated with $D_{n}(4000)=1.2$. Except for three sources, NGC 5347, NGC 5929, and NGC 5252, the $D_{n}(4000)$ strengths of most HBLR Seyfert 2 galaxies are smaller than those of non-HBLR galaxies. These three HBLR Seyfert 2 galaxies were previously classified as non-HBLR Seyfert 2 galaxies[2][13] and only found to show HBLRs with the Keck telescope[14][15]. Therefore, the HBLRs in these three sources are substantially weaker than those in the other HBLR Seyfert 2 galaxies. This implies that the visibility of HBLRs is related to $D_{n}(4000)$ strengths. Based on the simple stellar population models[16], the corresponding stellar age of $D_{n}(4000)=1.2$ is approximately $2.5 \times 10^{8}$ years. These results indicate that the stellar ages of the majority of the non-HBLR Seyfert 2 galaxies are older than several $10^{8}$ years. This observation is also consistent with the overabundance of nitrogen in the non-HBLR Seyfert 2 galaxies, which can be explained by the evolution of starburst activities in these Seyfert 2 galaxies[9].

The $D_{n}(4000)$ strength might be affected by the observed sizes or the Hubble types of the galaxies. Because the aperture of SDSS (3") is similar to the slit width of our observation (2.9"), we compared the distance distribution in our sample. The average distance of the HBLR and non-HBLR galaxies is 62.2 and $58.5 \mathrm{Mpc}$ respectively. Both the Kolmogorov-Smirnov test $(P$ $=9.1 \%)$ and Studentąes $T$-test $(P=16.3 \%)$ show that the distance distributions of the HBLR and non-HBLR Seyfert 2 galaxies are similar. Our results indicate that there is no apparent relation between distance and $D_{n}(4000)$ strength. These results are unlikely to have been caused by the aperture effect. We also investigate the effects of Hubble type of the host galaxies. The stellar ages around the nuclei would naturally be different if the HBLR and non-HBLR Seyfert 2 galaxies have different types of host galaxies. We use a numerical Hubble type proposed by De Vaucouleurs to compare the morphology of our samples. There is no relation between the Hubble type and the $D_{n}(4000)$ strength measured within the nuclear regions. Whether the host galaxies are early or 


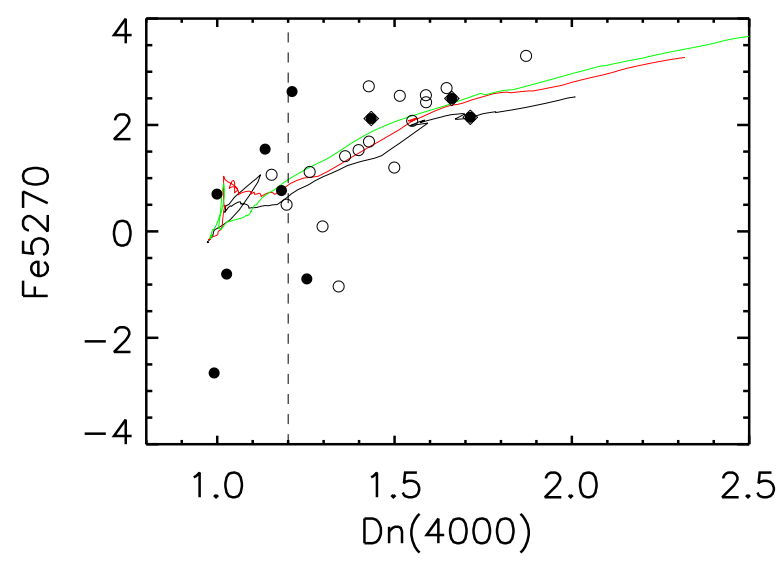

Figure 1: The $D_{n}(4000)$ strengths and the spectral index Fe5270 of the 10 HBLR and 16 non-HBLR Seyfert 2 galaxies. Symbols: filled circle - HBLR Sy2s; open circle - Non-HBLR Sy2; Diamonds - weak HBLRs: NGC 5347, NGC 5929, and NGC 5252. The lines show the evolution of the stand simple stellar population models[16] at ages from $10^{5}$ to $10^{10} \mathrm{yr}$ for the mentallicities (Black: $\mathrm{Z}=0.008$; Red: $\mathrm{Z}=0.02$; Green: $\mathrm{Z}=0.05)$. The $D_{n}(4000)$ strength of the non-HBLR Seyfert 2 galaxies is larger than that of HBLR galaxies, indicating that the stellar population of non-HBLR galaxies is older than that of HBLR galaxies.

late types, the $D_{n}(4000)$ strengths of the non-HBLR Seyfert 2 galaxies are larger than those of the HBLR galaxies.

Our results show that the stellar population of the non-HBLR Seyfert 2 galaxies is older than that of HBLR galaxies. Storchi-Bergmann et al. (2000)[17] and González Delgado et al. (2001)[18] have shown that nuclear starbursts might be a general phenomenon in Seyfert galaxies. Storchi-Bergmann et al. (2001)[19] further suggested that a composite Seyfert $2+$ starburst nucleus might evolve into a pure Seyfert 2 nucleus with an old stellar population. Our results suggest that the state of HBLR and non-HBLR galaxies might represent different evolutionary stages of star formation. We propose a potential evolutionary scenario for Seyfert galaxies. When certain mechanisms, such as nuclear bars[20] or merger processes transport material from outer regions to feed AGNs, gas accumulates in the nuclear regions and triggers star formation. During this period, galaxies could be dominated by AGNs as well as star formation activities (i.e., Seyfert 1 and HBLR Seyfert 2 galaxies). However, when the specified mechanisms stop transporting material, the power of AGNs declines and the BLRs might vanish. In the occurrence of this event, the nucleus then becomes a pure Seyfert 2 nucleus with an evolved stellar population (i.e., non-HBLR Seyfert 2 galaxies).

The scenario predicts the potential development of two different populations of non-HBLR Seyfert 2 galaxies. When Seyfert 1 and HBLR Seyfert 2 galaxies evolve into non-HBLR galaxies, the original orientations of the torus should remain preserved. In the other words, a Seyfert 1 nucleus evolve into a "face-on" non-HBLR nucleus, and a HBLR Seyfert 2 nucleus evolves into an "edge-on" non-HBLR Seyfert 2 nucleus. This suggests that some non-HBLR Seyfert 2 galaxies might actually have a face-on orientation without heavy obscuration. This prediction is consistent with the outcome of a hydrogen column density distribution of non-HBLR Seyfert 2 galaxies; taken 
from a large sample of 120 Seyfert 2 galaxies (49 HBLR and 71 non-HBLR) compiled by Wu et al. (2011)[4], we note that $40 \%$ of non-HBLR Seyfert 2 galaxies are unabsorbed; that is, $\mathrm{N}_{H}$ less than $10^{22} \mathrm{~cm}^{-2}$. Conversely, only $60 \%$ of non-HBLR Seyfert 2 galaxies are absorbed, whereas a higher fraction of HBLR (83\%) galaxies have a column density larger than $10^{22} \mathrm{~cm}^{-2}$. Tran et al. (2011)[8] discovered some "naked" non-HBLR Seyfert 2 galaxies that exude X-ray variability, but intrinsically lack BLRs. We suggest that these naked non-HBLR Seyfert 2 galaxies belong to the face-on non-HBLR galaxies.

\section{SUMMARY}

Overall, our results suggest that (1) the stellar population of the non-HBLR Seyfert 2 galaxies is older than HBLR galaxies, suggesting that there might be an evolutionary process; (2) the ages of nuclear region stellar populations is unrelated to the morphology of host galaxies; and (3) Seyfert 1 and HBLR Seyfert 2 galaxies might respectively evolve into unabsorbed and absorbed non-HBLR Seyfert 2 galaxies. The optical variability of the "face-on" non-HBLR Seyfert 2 galaxies might be detectable. This possibility can be tested in future observations.

\section{Acknowledgments}

We are grateful to the staffs of BOAO. This work was partially supported by the National Science Council of Taiwan (grants NSC 99-2112-M-008-014-MY3 (C.-Y.H.), NSC 100-2119-M008-011-MY3 (C.-Y.H.), NSC 99-2112-M-001-002-MY3 (K.Y.H.) and NSC 100-2112-M-001001-MY3 (Y.O.)). Funding for SDSS-III has been provided by the Alfred P. Sloan Foundation, the Participating Institutions, the National Science Foundation, and the U.S. Department of Energy Office of Science. The SDSS-III web site is http://www.sdss3.org/. SDSS-III is managed by the Astrophysical Research Consortium for the Participating Institutions of the SDSS-III Collaboration including the University of Arizona, the Brazilian Participation Group, Brookhaven National Laboratory, University of Cambridge, Carnegie Mellon University, University of Florida, the French Participation Group, the German Participation Group, Harvard University, the Instituto de Astrofisica de Canarias, the Michigan State/Notre Dame/JINA Participation Group, Johns Hopkins University, Lawrence Berkeley National Laboratory, Max Planck Institute for Astrophysics, Max Planck Institute for Extraterrestrial Physics, New Mexico State University, New York University, Ohio State University, Pennsylvania State University, University of Portsmouth, Princeton University, the Spanish Participation Group, University of Tokyo, University of Utah, Vanderbilt University, University of Virginia, University of Washington, and Yale University. This research has made use of the NASA/IPAC Extragalactic Database (NED) which is operated by the Jet Propulsion Laboratory, California Institute of Technology, under contract with the National Aeronautics and Space Administration.

\section{References}

[1] R. Antonucci, Unified Models for Active Galactic Nuclei and Quasars, ARA\&A, 1993 (31) 473

[2] H. D. Tran, The Unified Model and Evolution of Active Galaxies: Implications from a Spectropolarimetric Study, ApJ, 2003 (583) 632 
[3] C. A. Heisler, S. L. Lumsden, \& J. A. Bailey, Visibility of scattered broad-line emission in Seyfert 2 galaxies, Nature, 1997 (385) 700

[4] Y. Z. Wu, E. P. Zhang, Y. C. Liang, C. M. Zhang, \& Y. H. Zhao, The Different Nature of Seyfert 2 Galaxies With and Without Hidden Broad-line Regions, ApJ, 2011 (730) 121

[5] E. P. Zhang, \& J. M. Wang, The Unified Model of Active Galactic Nuclei. I. Non-Hidden Broad-Line Region Seyfert 2 and Narrow-Line Seyfert 1 Galaxies, ApJ, 2006 (653) 137

[6] P. C. Yu, \& C. Y. Hwang, Dust Morphology of Hidden Broad-Line Region and Non-Hidden Broad-Line Region Seyfert 2 Galaxies, ApJ, 2005 (631) 720

[7] X. W. Shu, J. X. Wang, P, Jiang, L. L. Fan, \& T. G. Wang, Investigating the Nuclear Obscuration in Two Types of Seyfert 2 Galaxies, ApJ, 2007 (657) 167

[8] H. D. Tran, J. E. Lyke, \& J. A. Mader, Indecent Exposure in Seyfert 2 Galaxies: A Close Look, ApJ, 2011 (726) L21

[9] P. C. Yu, \& C. Y. Hwang, Ionization Mechanisms of HBLR and Non-HBLR Seyfert 2 Galaxies, AJ, 2011 (142) 14

[10] M. L. Balgoh, S. L. Morris, H. K. C. Yee, R. G. Carlberg, \& E. Ellingson, Differential Galaxy Evolution in Cluster and Field Galaxies at $z \sim 0.3$, ApJ, 1999 (527) 54

[11] G. Kauffmann, G., et al., Stellar masses and star formation histories for $10^{5}$ galaxies from the Sloan Digital Sky Survey, MNRAS, 2003 (341) 33

[12] A. G. Bruzual, Spectral evolution of galaxies. I - Early-type systems, ApJ, 1983 (273) 105

[13] S. Young, J. H. Hough, A. Efstathiou, B. J. Wills, J. A. Bailey, M. J.Ward, \& D. J. Axon, Polarimetry and modelling of narrow-line active galaxies, MNRAS, 1996b (281) 1206

[14] E. C. Moran, A. J. Barth, L. E. Kay, \& A. V. Filippenko, The Frequency of Polarized Broad Emission Lines in Type 2 Seyfert Galaxies, ApJ, 2000 (540) L73

[15] H. D. Tran, Hidden Double-peaked Emitters in Seyfert 2 Galaxies, ApJ, 2010 (711) 1174

[16] G. Bruzual, \& S. Charlot, Stellar population synthesis at the resolution of 2003, MNRAS, 2003 (344) 1000

[17] T. Strochi-Bergmann, D. Raimann, E. L. D. Bica, \& H. A. Fraquelli, The Frequency of Nuclear Star Formation in Seyfert 2 Galaxies,ApJ, 2000 (544) 747

[18] R. M. González Delgado, T. Heckman, \& C. Leitherer, The Nuclear and Circumnuclear Stellar Population in Seyfert 2 Galaxies: Implications for the Starburst-Active Galactic Nucleus Connection, ApJ, 2001 (546) 845

[19] T. Strochi-Bergmann, R. M. González Delgado, H. R. Schmitt, R. C. Fernands, \& T. Heckman, Circumnuclear Stellar Population, Morphology, and Environment of Seyfert 2 Galaxies: An Evolutionary Scenario, ApJ, 2001 (559) 147

[20] I. Shlosman, J. Frank, \& M. C. Begelman, Bars within bars - A mechanism for fuelling active galactic nuclei, Nature, 1989 (338) 45 